\title{
EFECTO DEL NIM EN EL DAÑO OCASIONADO POR EL GUSANO COGOLLERO SPODOPTERA FRUGIPERDA (SMITH) (LEPIDOPTERA: NOCTUIDAE) EN TRES VARIABLES AGRONÓMICAS DE MAÍZ RESISTENTE Y SUSCEPTIBLE
}

\section{Socorro del Carmen GuTIÉRREZ-GARCÍA1, Julio SÁNCHEZ-ESCUDERO1, Juan Francisco PÉrez-Domínguez ${ }^{*}$, Aquiles Carballo-Carballo², David BergVinson ${ }^{4}$ y María Martha AguILERA-PEÑA ${ }^{5}$}

${ }^{1}$ Instituto de Fitosanidad (IFIT) Colegio de Postgraduados. Montecillo, Texcoco Edo. de México, MÉXICO.

2 Instituto de Recursos Genéticos y Productividad (IREGEP) Colegio de Postgraduados. Montecillo, Texcoco, Edo. de México, MÉXICO.

3 INIFAP. Campo Experimental Centro Altos de Jalisco. Apartado Postal 79 C.P. 47800 Ocotlán, Jalisco, MÉXICO. E-mail: fcopedom@yahoo.com

4 Centro Internacional para el Mejoramiento del Maíz y el Trigo (CIMMYT) Programa de Maíz. El Batán, Texcoco, Edo. de México, MÉXICO.

5Dirección General de Sanidad Vegetal. Consejo Nacional Consultivo Fitosanitario. Colegio de Postgraduados. Montecillo, Texcoco, Edo. de México, MÉXICO.

* Autor para correspondencia.

Gutiérrez-García, S. del C., J. Sánchez-Escudero, J. F. Pérez-Domínguez, A. Carballo-Carballo, D. Bergvinson \& M. M. Aguilera-Peña. 2010. Efecto del nim en el daño ocasionado por el gusano cogollero Spodoptera frugiperda (Smith) (Lepidoptera: Noctuidae) en tres variables agronómicas de maíz resistente y susceptible. Acta Zoológica Mexicana (n.s.), 26(1): 1-16.

RESUMEN. En dos experimentos realizados durante dos ciclos de cultivo de maíz, se evaluó el efecto de un formulado de aceite de nim Azadirachta indica A. Juss en larvas de gusano cogollero Spodoptera frugiperda (J. E. Smith). El objetivo fue conocer el daño ocasionado por el gusano cogollero en plantas de maíz resistente (CML-67) y maíz susceptible (CML-131) ambas tratadas con nim, en campo. En un primer experimento se evaluaron épocas de aplicación del aceite de A. indica en dos líneas de maíz, una resistente (CML-67) y otro susceptible (CML-131) a $S$. frugiperda cultivados en Poza Rica, Veracruz, México. El aceite en concentración de 20,8\%, se aplicó sobre las hojas superiores y cogollo, con los siguientes tratamientos: cuatro horas, antes de la infestación artificial con larvas de primer ínstar, 7 y 14 días después de la infestación; se incluyó un tratamiento con el insecticida químico Permetrina y un testigo absoluto (sólo agua). En el segundo experimento, sobre las mismas dos líneas de maíz, CML-67 y CML-131 se aplicaron las concentraciones $20,8,2,0$ y 0,20\% de aceite de $A$. indica. Cada tratamiento se aplicó tres veces sin intervalo fijo durante la etapa vegetativa del cultivo, se incluyó al insecticida Permetrina y al testigo absoluto. Se registró el daño foliar mediante la escala estandarizada de daño de Mihm del 0 al 9, altura de la planta y el rendimiento. En las fechas de aplicación del nim, el daño foliar 
de la línea susceptible osciló de 6,7 a 8,1 en promedio, según la escala de Mihm, representó alto daño y en la línea resistente, daño foliar moderado $(4,6$ a 6,0). En el experimento de concentraciones de nim, en el ciclo I se observaron daños de 4,4 a 6,2 en promedio y en el ciclo P-V los daños fueron de 6,5 a 8,5 en ambas líneas. La floración no presentó diferencias significativas en las diferentes fechas de aplicación, mientras que en el experimento de diferentes concentraciones el tratamiento $20,8 \%$ de aceite de $A$. indica se adelantó 7,4 días con respecto al testigo. No se encontraron diferencias al cuantificar la altura. No obstante para el tratamiento con $20,8 \%$ de aceite de $A$. indica la defoliación fue menor y no se obtuvieron buenos rendimientos, se apreció que a esta concentración de aceite, A. indica causó fitotoxicidad. El nim permitió mayor protección cuando se aplicó antes de que se presentara la plaga a concentraciones menores al $2 \%$ y se reforzó con posteriores aplicaciones a intervalos de una aplicación por semana.

Palabras clave: Desarrollo biológico, fitotoxicidad nim, Azadirachta indica, maíz, Spodoptera frugiperda.

Gutiérrez-García, S. del C., J. Sánchez-Escudero, J. F. Pérez-Domínguez, A. Carballo-Carballo, D. Bergvinson \& M. M. Aguilera-Peña. 2010. Effect of Neem on damage caused by fall armyworm Spodoptera frugiperda (Smith) (Lepidoptera: Noctuidae) and three agricultural variables on resistant and susceptible maize.Acta Zoológica Mexicana (n.s.), 26(1): 1-16.

ABSTRACT. Neem oil was evaluated against fall armyworm Spodoptera frugiperda (J. E. Smith) on plants of two maize lines, one resistant and another one susceptible, planted in Poza Rica, Veracruz, México. The objective of this study was to compare biological development of resistant and susceptible maize plants infested by fall armyworm and sprayed with neem oil under field conditions. A concentration of $20,8 \%$ neem oil, was applied on the upper leaves and whorl according to the following treatments: before artificial infestation, one and two weeks after infestation; in addition, a chemical insecticide (Permetrin) and one control (water) were included. In an experiment comparing different doses, neem was applied 3 times at concentrations of 20,8,2,0 and 0,20\%; with a Permetrin and water control included for comparison. Damage to foliage, flowering, plant height, and yield were then recorded. In the experiment to determine time of application the susceptible line showed severe damage from 6,7 to 8,1 on a scale from $0-9$. while the resistant line exhibited damage ranging from 4,6 to 6,0. For the neem oil dose experiment, plants in both lines during the winter cycle showed damage ranging from 4,4 to 6,2 , while during the spring-fall season damage was from 6,2 to 8,5. Flowering was not affected by application dates. However in the experiment with different neem oil concentrations, for the $20,8 \%$ treatment flowering was accelerated up to 7,4 days when compared to the control. No differences were found among treatments on in plant height. The treatment with $20,8 \%$ neem oil showed low defoliation, but yields were low at this high doses due to phytotoxicity. Weekly application of $2 \%$ neem oil gave more than best protection when applied before pest infestations.

Key words: biological development, neem phytotoxicity: Azadirachta indica, maize, Spodoptera frugiperda.

\section{INTRODUCCIÓN}

El nim Azadirachta indica A. Juss. (Meliaceae) contiene en sus semillas, tallo y hojas a la azadiractina, sustancia con efectos antialimentarios y repelentes a insectos plaga de cultivos agrícolas (Levin-Mitchell et al. 2004) su amplia gama de acción en aproximadamente 200 especies plaga de importancia agrícola, doméstica, médica y veterinaria (Mulla y Su, 1999; Levin-Mitchell et al. 2004), en diversos cultivos y 
zonas agrícolas ha estimulado su uso e investigación (Schmutterer 1990; Yakkundi et al. 1996) siendo el insecticida vegetal más estudiado (Mulla \& Su, 1999) y utilizado como polvo, extracto acuoso y etanólico o como aceite. El producto más usado es el extracto acuoso de la semilla (Levin-Mitchell et al. 2004).

El nim, al igual que la gran mayoría de insecticidas vegetales, no provoca mortalidad rápida como los insecticidas organosintéticos, por lo que las metodologías y técnicas de evaluación deben de encaminarse a probar el efecto insectistático (Schmutterer 1990). Aunque ha sido reportada la actividad del nim contra plagas de importancia agrícola, como Ostrinia nubilalis, Spodoptera litura, Plutella xylostella,Clavigralla scutellaris entre otras (Levin Mitchell et al., 2004), no hay reportes de trabajos realizados dirigidos a integrar el nim con otros métodos de control, como la resistencia vegetal a insectos.

La presente investigación tuvo como objetivo: conocer el daño ocasionado por el gusano cogollero Spodoptera frugiperda y las variaciones en floración, altura de planta y rendimiento en maíz resistente (CML-67) con nim y maíz susceptible (CML-131) con nim, en campo.

\section{MATERIALES Y MÉTODOS}

La investigación se realizó en el Campo Experimental del Centro Internacional para el Mejoramiento del Maíz y Trigo (CIMMYT) en Poza Rica, Veracruz, México, durante los ciclos de invierno 1998-1999 (22 de diciembre al 12 de mayo) y primavera-verano 1999 (16 de junio al 22 de octubre), en condiciones de riego.

Preparación del terreno. Se inició con una labor de rastra para incorporar la cobertura vegetal Crotalaria spp. y Mucuna gigantea (Flowers) (Leguminosae), enseguida fue removido el suelo con el implemento "pequeño gigante", posteriormente se le dieron dos rastreos cruzados para nivelar el terreno y una aplicación de fertilizante NPK a una concentración de 200-80-00, finalmente se surcó y bordeó.

La siembra de las líneas de maíz CML-67 y CML-131, proporcionadas y catalogadas como resistente (por antibiosis y tolerancia) y susceptible, respectivamente, por el CIMMYT (Kumar, 1994), se realizó el 22 de diciembre del 1997 para el ciclo de invierno y el 16 de junio del 1998 para el ciclo de primaveraverano. Se sembraron 20 semillas de cada línea de maíz por surco, alternando 2 surcos de la línea resistente y 2 surcos de la susceptible para tener un total de 20 surcos (10 de CML-67 y 10 de CML-131), en un terreno de $16 \mathrm{~m}$ de ancho y $20 \mathrm{~m}$ de largo para cada uno de los experimentos. En cada unidad experimental hubo 20 plantas de maíz por tratamiento a una distancia de $25 \mathrm{~cm}$ entre plantas y $80 \mathrm{~cm}$ entre surcos. Al terminar la siembra, se realizó el tapado de las semillas y la aplicación de 7 1/ha del herbicida Primagram 500 FW (mezcla de metolaclor 23,3\% con atrazina 21,9\%), Ciba-Geigy Mexicana, S. A. de C. V. 
Infestación artificial con larvas de $\boldsymbol{S}$. frugiperda. Para asegurar altas densidades del gusano cogollero en los experimentos, se realizaron infestaciones artificiales con larvas de primer instar a los 50 días posteriores a la siembra. Las larvas fueron depositadas directamente al cogollo de cada planta mediante una "bazuka" (Mihm, 1989), diseñada para expulsar en promedio $35 \pm 5$ larvas por cogollo cuando las plantas tuvieron dos hojas liguladas y altura entre 20 a $25 \mathrm{~cm}$; las infestaciones artificiales fueron realizadas entre 8:45 y 9:45 a. m.

Las larvas de primer instar de $S$. frugiperda utilizadas en esta investigación se obtuvieron de la cría masiva de insectos que mantiene el CIMMYT, en el Laboratorio de Entomología de El Batán, Texcoco, México. En cada fecha de infestación las larvas fueron transportadas al Campo Experimental de Poza Rica, Veracruz, en una hielera para evitar su deshidratación. A las larvas se les adicionaron pedazos de olote, para facilitar su manipulación durante el proceso de infestación.

Aplicación del nim. El aceite de nim utilizado fue el NEEM OIL EXTRACT ${ }^{\circledR}$ (93\%), con una concentración de azadiractina de 0,4463\%, obtenido en la Consultoría Integral P.S., S.A. de C.V., México. Entre las 9:30 a las 10 a.m., el aceite se asperjó con un atomizador manual de $1000 \mathrm{ml}$ de capacidad, sobre el cogollo y hojas superiores de cada una de las 80 plantas de cada tratamiento.

Tratamientos. En un experimento se prepararon tres concentraciones del aceite de nim siguientes: $20,8 \%, 2,0 \%$ y $0,2 \%(67,2,6,72$ y $0,672 \mathrm{~mL}$ de producto en $300 \mathrm{~mL}$ de agua, respectivamente). Se utilizaron dos testigos de comparación; uno con agua potable y el tratamiento convencional con Permetrina 0,4\% (POUNCE ${ }^{\circledR} 4 \mathrm{G}$ FMC Agroquímica de México S. de R.L. de C.V.).

La época y las concentraciones de aplicación de nim conformaron dos experimentos. En el de épocas de aplicación de nim se utilizó la concentración de 20,8\%, aplicada una sola vez en 3 fechas diferentes: $4 \mathrm{~h}$ antes de la infestación artificial con $S$. frugiperda (IA), 7 y 14 días después de la IA (50, 54 y 67 días después de la siembra, respectivamente). El tratamiento POUNCE ${ }^{\circledR}$ (Permetrina 0,4\% FMC Agroquímica de México S. de R.L. de C.V.) solo se aplicó una semana después de la IA (57 días después de la siembra), aplicando 0,25 g /cogollo; mientras que el agua se asperjó cada vez que se aplicó el aceite de nim.

En el experimento de concentraciones $(20,8,2,0$ y $0,2 \%$ de aceite de nim) se aplicaron 3 veces en cada fecha ( $4 \mathrm{~h}$ antes de la IA , 7 y 14 días después de la IA). En sendos experimentos se utilizaron los dos testigos de comparación: el insecticida convencional y el agua. Las aspersiones de nim en el cogollo de cada una de las plantas fueron realizadas cuando el cultivo estaba en la etapa de 5 a 6 hojas ( 2 a 3 hojas liguladas), en las fechas y dosis establecidas para cada tratamiento. 
Diseño experimental. En el terreno experimental, de 20 surcos con 40 plantas cada uno, los primeros trazos consistieron en crear a lo largo del surco cuatro bloques, que se constituyeron con 10 parcelas cada uno; definiendo a la parcela experimental de 2 surcos, con 10 plantas cada uno. De modo que en cada bloque se ubicaron alternadamente 5 parcelas con la línea susceptible y 5 parcelas con la línea resistente. Las unidades experimentales representaron las parcelas chicas en un diseño de bloques al azar con arreglo en parcelas divididas. Las parcelas grandes fueron los cinco tratamientos que consistieron en 3 dosis de aceite de nim, testigo químico y testigo absoluto, considerados para cada experimento en los dos ciclos agrícolas. Cada tratamiento tuvo cuatro repeticiones en cada experimento.

\section{Variables evaluadas}

Daño. La defoliación de $S$. frugiperda se cuantificó por planta utilizando la escala de daño propuesta por Mihm (1983), considerando como 0 al daño ligero con pequeñas perforaciones y 9 a la planta muerta. Se efectuaron tres registros de daño para cada tratamiento: el 10 de febrero (7 DDIA), el 17 de febrero (14 DDIA) y el 10 de marzo (28 DDIA) para el ciclo de invierno, y el 16 de julio (7 DDIA), el 22 de julio (14 DDIA) y el 6 de agosto (28 DDIA) para el ciclo primavera-verano.

Floración. El tiempo de la floración de maíz se registró en el momento en que se encontraba floreciendo el $50 \%$ de las plantas de cada surco, de modo que por cada parcela se realizaron 2 tiempos de floración.

Altura de planta. La altura de la planta se midió cuando se presentó el 50\% de floración. Se midió en la primera planta de cada surco, de la base de la planta a la base de la lámina de la última hoja formada.

Rendimiento. Se registró en peso seco el rendimiento de maíz (mazorca sin glumas) por parcela a los 141 días después de la siembra para el ciclo de invierno y a los 128 días después de la siembra para el ciclo primavera-verano.

Se obtuvo información de la temperatura ambiental y la precipitación pluvial mediante registros de una estación climatológica.

Análisis estadístico. los datos de porcentaje de daños fueron transformados a arcosen para facilitar el análisis, debido a que de la manera como fueron tomados no hay ajuste a una distribución normal. A los resultados de nivel de daño, tiempo de floración, altura y rendimiento se les aplicó un análisis de varianza para un diseño de bloques completos al azar con arreglo en parcelas divididas y en las variables donde hubo diferencias significativas se realizó la prueba de rango múltiple de Tukey ( $\alpha=$ $0,05)$ para la comparación de medias, utilizando el paquete estadístico SAS (SAS Institute, 2000). 


\section{RESULTADOS}

Fechas de aplicación del aceite de $\boldsymbol{A}$. indica. El daño foliar causado por $S$. frugiperda en los tres muestreos fue similar estadísticamente en todos los tratamientos durante el ciclo PV incluyendo el testigo con insecticida y el testigo absoluto, osciló de 5,1 a 8,1. En el ciclo I el daño fue menor, lo que indica que en P-V el daño foliar fue 14,5\% mayor (Figs. 1, 2 y 3, 7,8 y 9). En esta variable el nim fue efectivo sobre el gusano cogollero únicamente a los 7 días posteriores a la IA y las observaciones del daño foliar realizadas a los 14 y 28 días indicaron que el nim no afectó el desarrollo biológico del gusano cogollero (Figs. 2 y 3). Durante el ciclo I la aplicación de aceite de nim antes de la infestación del gusano cogollero en la línea resistente presentó los más bajos índices de daño ( 4,31 en promedio) aunque no hay diferencia significativa entre los demás tratamientos de nim y el testigo absoluto, solamente el testigo químico fue estadísticamente diferente (Fig. 3).

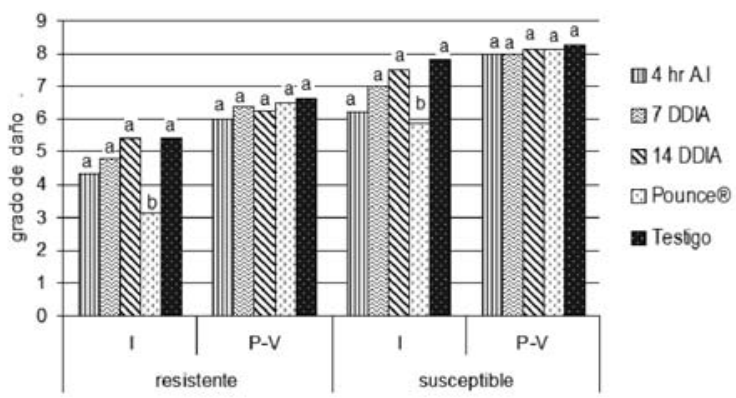

Figura 1. Grado de daño de gusano cogollero, 7 días después de la infestación, durante 1998. $\mathrm{AI}=$ Antes de la Infestación. $\mathrm{I}$ = invierno; PV = primavera verano; DDIA Días después de la infestación artificial. Tratamientos con la misma letra en las columnas no son significativamente diferentes según Tukey $(\alpha \leq 0.05)$

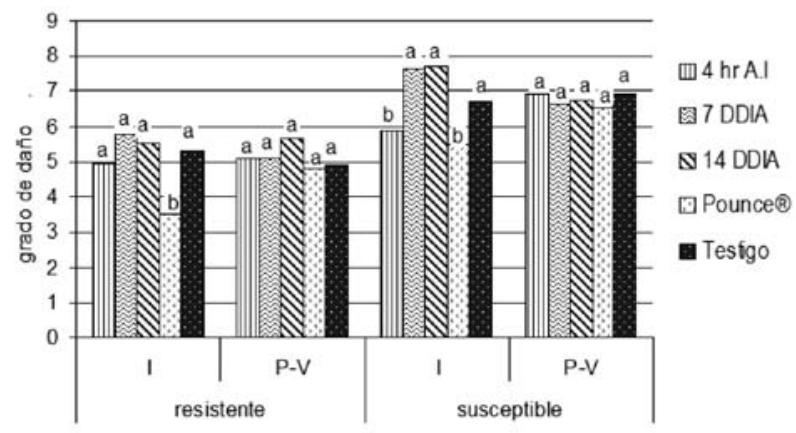

Figura 2. Grado de daño de gusano cogollero, 14 días después de la infestación, durante 1998. $\mathrm{AI}=$ Antes de la Infestación. I = invierno; PV = primavera verano; DDIA Días después de la infestación artificial. Tratamientos con la misma letra en las columnas no son significativamente diferentes según Tukey $(\alpha \leq 0.05)$ 


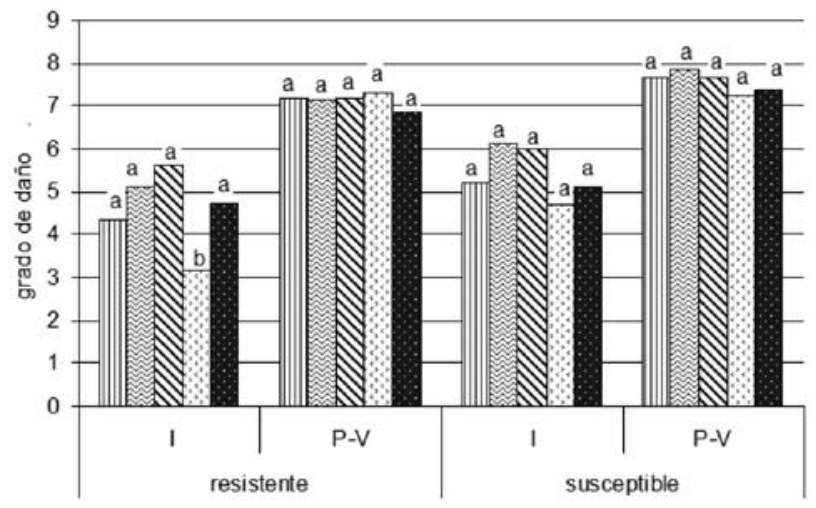

\section{띠 $4 \mathrm{hr}$ A.I \\ 园 $7 D D A$ \\ \14 DDA \\ [.] Pounce@ \\ Tesigo}

Figura 3. Grado de daño de gusano cogollero, 14 días después de la infestación, durante 1998.

AI=Antes de la Infestación. I = invierno; PV = primavera verano; DDIA Días después de la infestación artificial. Tratamientos con la misma letra en las columnas no son significativamente diferentes según Tukey $(\alpha \leq 0.05)$

En lo que respecta al efecto de las fechas de aplicación del aceite de nim en la floración (Fig. 4), analizando ciclos ésta requirió de 81,3 a 113,1 días en los dos ciclos; en el ciclo I fue más tardía con 97,3 días en promedio, a diferencia del ciclo P-V, esto fue más evidente en la línea susceptible. Con relación en las líneas, la floración en la línea resistente se retrasó en 5,7 días en ambos ciclos, en tanto que en la línea susceptible se atrasó 31,8 días. Los datos de floración de los tratamientos fueron similares al testigo, excepto en el tratamiento con aplicación realizada 4 horas antes de la infestación de $S$. frugiperda, que a pesar de no ser estadísticamente diferente del testigo e insecticida se adelantó 2 días en promedio para ambas líneas con respecto al testigo (Fig. 4).

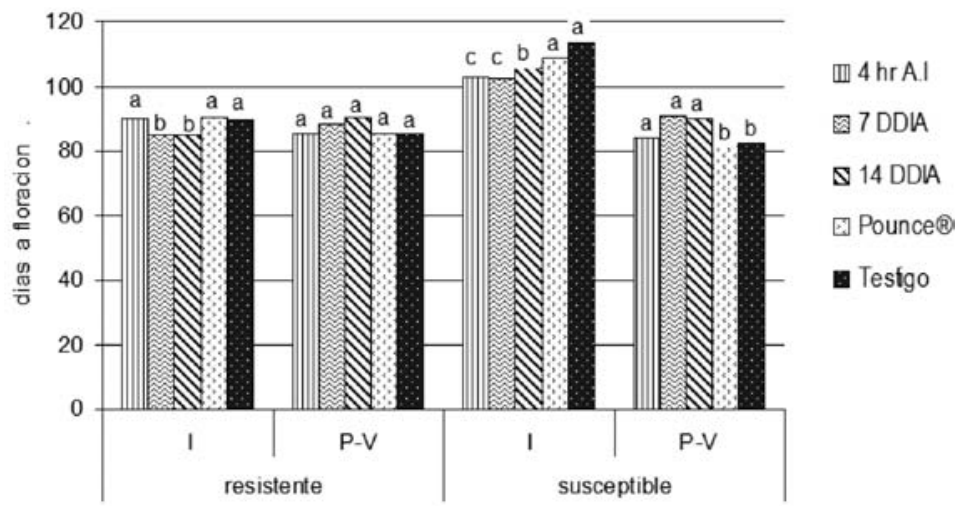

Figura 4. Días a floración de líneas de maíz resistente y susceptible, durante 1998.

AI=Antes de la Infestación. I = invierno; PV = primavera verano; DDIA Días después de la infestación artificial. Tratamientos con la misma letra en las columnas no son significativamente diferentes según Tukey $(\alpha \leq 0.05)$ 
La altura de planta fue mayor en el ciclo P-V en comparación al ciclo de I (Fig. 5). La línea de maíz susceptible fue más alta en $12,8 \mathrm{~cm}$ en promedio con respecto de la línea resistente; hubo diferencias significativas entre tratamientos en la altura de planta durante el ciclo P-V en ambas líneas y se observaron plantas con menor altura cuando el aceite de nim fue aplicado a los 14 DDIA (Fig. 5).

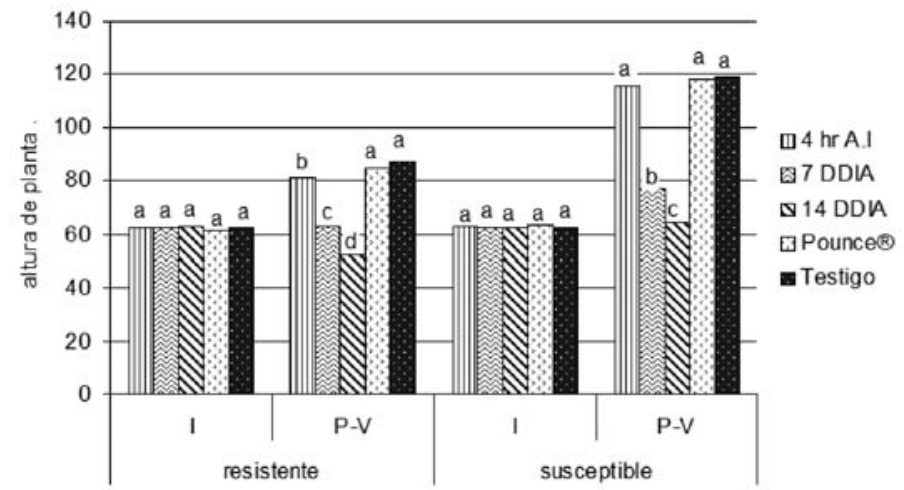

Figura 5. Altura de planta en líneas de maíz resistente y susceptible.

$\mathrm{AI}=$ Antes de la Infestación. $\mathrm{I}$ = invierno; PV = primavera verano; DDIA Días después de la infestación artificial. Tratamientos con la misma letra en las columnas no son significativamente diferentes según Tukey $(\alpha \leq 0.05)$

Con relación al rendimiento de grano (Fig. 6), en las líneas el rendimiento en la resistente fue de $964,8 \mathrm{~kg}^{\text {ha-1 }}{ }^{1}$ a diferencia de la línea susceptible con $316,6 \mathrm{~kg}^{\mathrm{ha}}{ }^{-1}$. Los rendimientos fueron mayores en I, con respecto a P-V en la mayoría de las mediciones Los mas bajos rendimientos fueron al aplicar el nim 14 días después de la IA, seguido por la aplicación de nim 7 días después de la IA, lo cual indica que aún en el genotipo resistente, el gusano cogollero causó un daño de importancia económica en los diversos tratamientos y que a ese nivel de infestaciones de larvas o después de ese tiempo, la aplicación de nim no reduce drásticamente las poblaciones de cogollero sino que el daño continúa.

En cuanto al efecto de los tratamientos en el rendimiento (Fig. 6), los tratamientos con aceite de $A$. indica tuvieron $54,1 \%$ menor rendimiento que el testigo, $844 \mathrm{~kg}$ ha- 1 en promedio. La aplicación de A. indica a la concentración de $20,8 \%$ realizada antes de la IA, 7 y 14 DDIA no protegió adecuadamente al maíz del daño de $S$. frugiperda, no proporcionó un efecto aditivo, ni potenció la protección con maíz resistente (Fig. 6).

Concentraciones con aceite de $\boldsymbol{A}$. indica. No hubo diferencias significativas respecto al daño foliar causado por $S$. frugiperda en los tres muestreos durante el ciclo P-V, para ambas líneas de maíz (Figs. 7, 8 y 9). En cambio, en el ciclo I a la 
concentración de $20,8 \%$ se detectaron diferencias en los primeros dos muestreos (hasta los 14 días), donde se observaron menos daños. El daño foliar causado por $S$. frugiperda, en P-V osciló de 6,5 a 7,3 a diferencia del ciclo I, donde varió de 4,5 a 6,0 . En I se redujo la defoliación de $S$. frugiperda en $21 \%$ (Figs. 7, 8 y 9). Se observó que el daño en la línea resistente fue menor que en la línea susceptible en 13,9\% y el promedio de daño en la línea resistente en P-V fue de 6,7 (daño severo) y en I de 4,6 (daño moderado). El daño promedio en la línea susceptible en P-V y en I indican que el daño fue severo en ambos ciclos y se observó una reducción del daño foliar de $18,9 \%$ en el ciclo I con respecto al ciclo P-V. Al comparar los resultados de daño en los tres muestreos en ambos ciclos la reducción del daño foliar en la línea resistente fue de $4,4 \%$ con respecto a la línea susceptible.

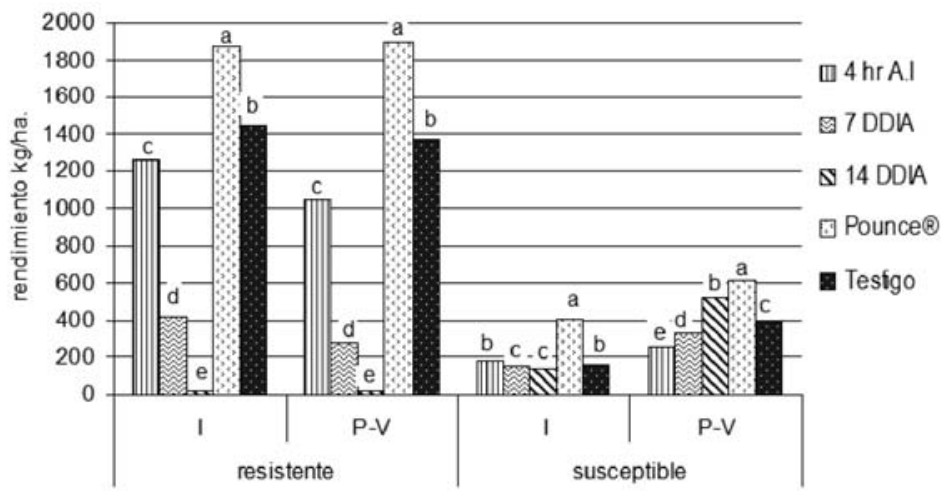

Figura 6. Rendimiento de grano en líneas de maíz resistente y susceptible, durante 1998.

AI=Antes de la Infestación. I = invierno; PV = primavera verano; DDIA Días después de la infestación artificial. Tratamientos con la misma letra en las columnas no son significativamente diferentes según Tukey $(\alpha \leq 0.05)$

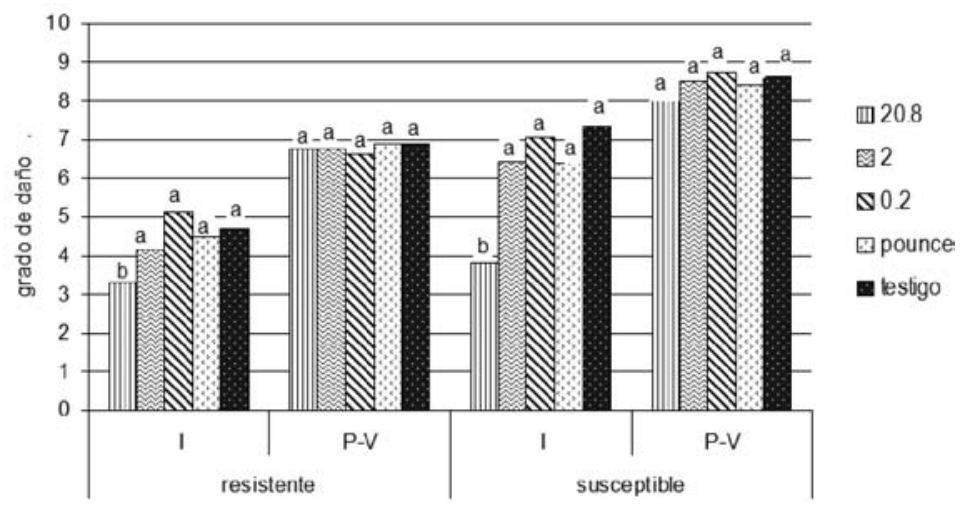

Figura 7. Grado de daño de gusano cogollero, 7 días después de la infestación. Durante 1999. AI=Antes de la Infestación. I = invierno; PV = primavera verano; DDIA Días después de la infestación artificial. Tratamientos con la misma letra en las columnas no son significativamente diferentes según Tukey $(\alpha \leq 0.05)$ 


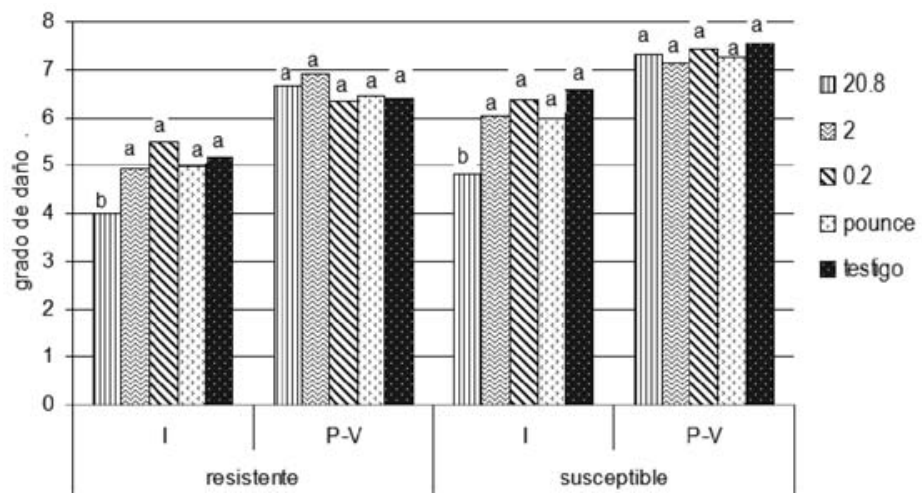

Figura 8. Grado de daño de gusano cogollero, 14 días después de la infestación durante 1999. AI=Antes de la Infestación. I = invierno; PV = primavera verano; DDIA = Días después de la infestación artificial. Tratamientos con la misma letra en las columnas no son significativamente diferentes según Tukey $(\alpha \leq 0.05)$

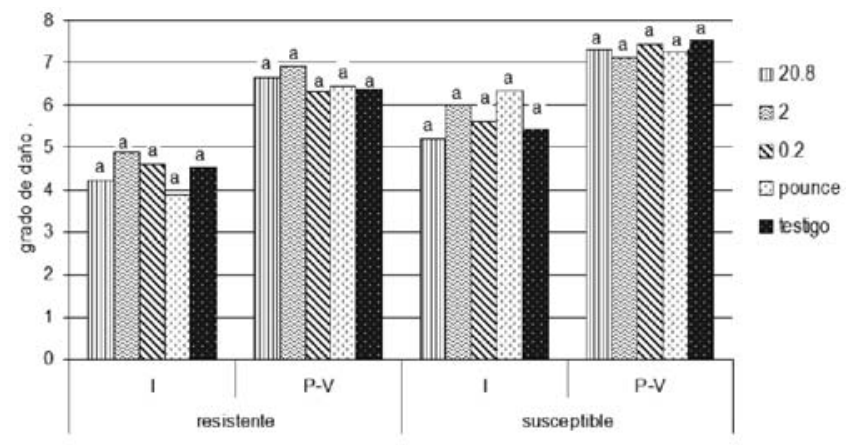

Figura 9. Grado de daño de gusano cogollero, 28 días después de la infestación durante 1999. AI=Antes de la Infestación. I = invierno; PV = primavera verano; DDIA = Días después de la infestación artificial.Tratamientos con la misma letra en las columnas no son significativamente diferentes según Tukey $(\alpha \leq 0.05)$

Con relación al daño foliar en las diferentes concentraciones evaluadas de aceite de $A$. indica, éstas fueron similares al testigo, excepto en la concentración de 20,8\% de aceite de $A$. indica (5,7 en promedio en la escala de daño), en la cual disminuyó en $8,3 \%$ el daño foliar en comparación al testigo (promedio de 6,4). Lo que sugiere en general que el aceite de nim en al menos dos de las concentraciones $(0,2$ y $2 \%)$ no protegieron adecuadamente durante todo el período al cogollo de las dos líneas de maíz del ataque de $S$. frugiperda.

En el caso de la altura de la planta (Fig. 11), en ambos ciclos varió de 81 a 117,1 $\mathrm{cm}$, mostrando mayor altura en el ciclo $\mathrm{P}-\mathrm{V}$ con un promedio de $101,5 \mathrm{~cm}$, el cual obtuvo $15,5 \mathrm{~cm}$ más que el ciclo I $(86 \mathrm{~cm})$ destacando el tratamiento $20,8 \%$ de aceite 
de nim. En relación con las líneas, la altura promedio en la línea susceptible fue de $99 \mathrm{~cm}$ y de $88,5 \mathrm{~cm}$ en la resistente, esto debido a que la línea resistente es generalmente de porte más bajo. Las plantas tratadas con el insecticida mostraron tres $\mathrm{cm}$ más de altura en promedio en comparación con el testigo.

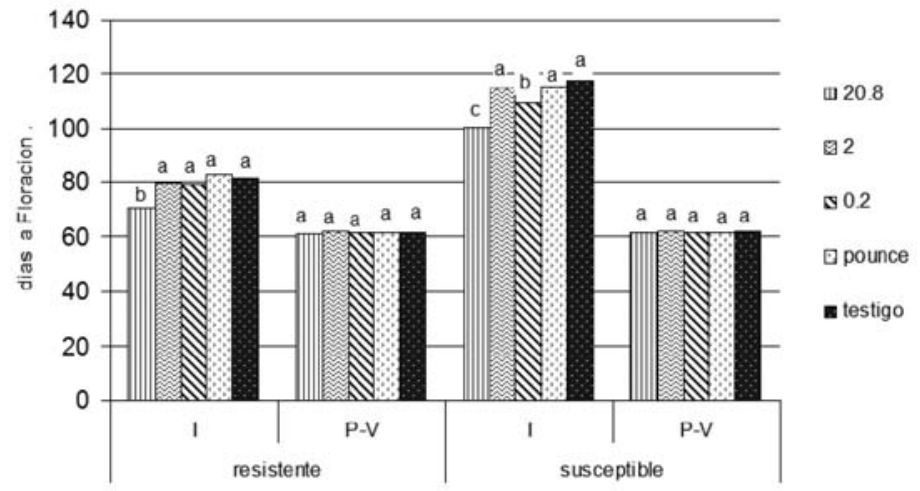

Figura 10. Días a floración de líneas de maíz resistente y susceptible, durante 1999.

$\mathrm{AI}=$ Antes de la Infestación. $\mathrm{I}$ = invierno; PV = primavera verano; DDIA Días después de la infestación artificial. Tratamientos con la misma letra en las columnas no son significativamente diferentes según Tukey $(\alpha \leq 0.05)$

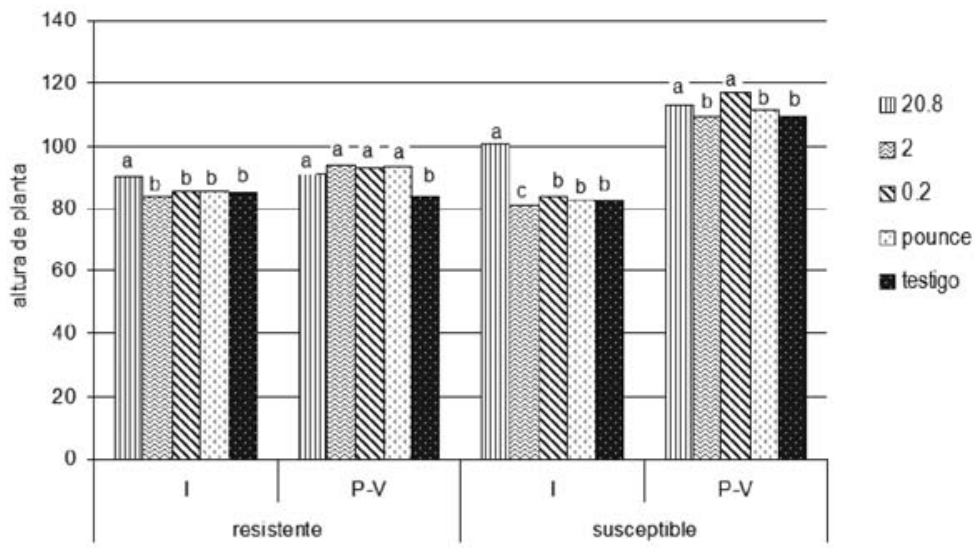

Figura 11. Altura de planta en líneas de maíz resistente y susceptible.

$\mathrm{AI}=$ Antes de la Infestación. $\mathrm{I}$ = invierno; PV = primavera verano; DDIA Días después de la infestación artificial. Tratamientos con la misma letra en las columnas no son significativamente diferentes según Tukey $(\alpha \leq 0.05)$ 
El rendimiento (Figs. 6 y 12), en general osciló de 102 a 1739,3 kg ha-1, resaltando el ciclo $\mathrm{P}-\mathrm{V}$ con $812,4 \mathrm{~kg}^{\mathrm{h}} \mathrm{-}^{-1}$ en promedio, mientras que en el ciclo I se cosecharon 705,2 kg ha-1. El rendimiento promedio de la línea resistente en ambos ciclos fue similar, $1133,5 \mathrm{~kg}$ ha-1 en P-V y $1131,1 \mathrm{~kg}$ ha-1 en I; en cambio en la línea susceptible el rendimiento promedio en entre ciclos fue variable. En I, hubo un incremento de $747,1 \mathrm{~kg}$ ha- ${ }^{-1}$ en promedio de la línea resistente con respecto a la línea susceptible. En cuanto al rendimiento promedio en las diferentes concentraciones de aceite de nim, éste fue de 13,5 y 39,2\% más de rendimiento que el testigo $(713,3 \mathrm{~kg}$ ha-1). Esta producción promedio contrasta con la obtenida en la concentración 20,8\% de aceite de A. indica, la cual fue menor en $36,8 \%$ en comparación con el testigo. El rendimiento promedio del tratamiento con el insecticida (1016,2 kg ha-1) mostró un incremento de $42,5 \%$ más que el testigo.

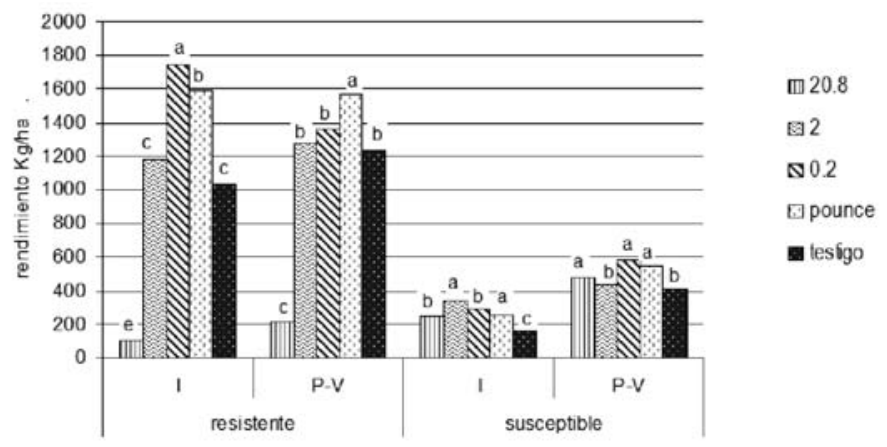

Figura 12. Rendimiento de grano en líneas de maíz resistente y susceptible, durante 1999. $\mathrm{AI}=$ Antes de la Infestación. I = invierno; PV = primavera verano; DDIA Días después de la infestación artificial. Tratamientos con la misma letra en las columnas no son significativamente diferentes según Tukey $(\alpha \leq 0.05)$

\section{DISCUSIÓN}

Fechas de aplicación del aceite de $\boldsymbol{A}$. indica. En el daño de gusano cogollero ocasionado al maíz tanto en el ciclo de I como en el de P-V, una aplicación de nim al 20,8\%, a los 50, 57 o 64 días después de la siembra, no protegió al maíz (susceptible o resistente) del daño de $S$. frugiperda. En el ciclo P-V, todas las concentraciones con aceite fueron estadísticamente iguales entre si, en ambas líneas de maíz. En el ciclo I la concentración 20,8\% tuvo menor daño hasta los primeros 14 DDIA, sobre todo en la línea resistente (CML-67). Se redujo el daño por defoliación del gusano cogollero en $21 \%$ en comparación al ciclo P-V.

El daño ocasionado por $S$. frugiperda en las líneas de maíz, en la resistente varió de 5,12 a 5,9 lo que se considera daño moderado, y en la susceptible fue daño severo, con registros de 6,5 a 7,5. El maíz resistente tuvo $15.9 \%$ menos daño foliar que el 
maíz susceptible. Esto concuerda con lo planteado por Williams y Davis (1997) y Williams et al. (1998) quienes encontraron que las variedades resistentes de maíz presentan un daño por $S$. frugiperda considerado como moderado o intermedio. El daño registrado en las tres épocas de aplicación (4 h antes de IA, 7 y 14 DDIA) no fue estadísticamente diferente del testigo absoluto en la mayoría de las parcelas, lo que indica que $A$. indica no protegió al maíz del daño de $S$. frugiperda.

Una aplicación de nim al 20,8\% tanto en I como en PV a los 50, 57 o 64 días después de la siembra no protegió al maíz (susceptible o resistente) del daño de $S$. frugiperda, de los 50 a los 78 días después de la siembra. La nula protección de nim se debió posiblemente a la alta temperatura, que osciló entre 25 a $30^{\circ} \mathrm{C}$ y de 35 a $40^{\circ} \mathrm{C}$ en ambos experimentos, lo que contribuyó al mejor desarrollo de $S$. frugiperda en consecuencia a un mayor daño al maíz y a la degradación de los compuestos naturales de A. indica evitando su eficacia en la protección al maíz, en concordancia con Yakkundi et al., (1996), Iannacone y Murrugarra (2002), Premachandra et al. (2005) quienes señalan que el aceite de $A$. indica sufre cambios químicos al ser expuesto a temperaturas mayores a $35{ }^{\circ} \mathrm{C}$. Asimismo, la azadiractina, al ser una molécula sensitiva, puede sufrir cambios químicos en la presencia de luz, temperatura y solventes orgánicos y por la exposición a los rayos de luz ultravioleta (Yakkundi et al. 1996; Stocke y Redfern 1982).

Otro factor que pudo influir en que la efectividad del nim haya durado solo duró 7 días fueron las lluvias que pudieron ocasionar la hidrolización del nim. Al respecto, según Levin-Mitchell et al. (2004) el efecto del nim dura desde 3 días hasta 2 semanas máximo, dependiendo de las condiciones climáticas y de la concentración de nim utilizados. En el presente estudio no se pudo cuantificar este efecto debido a falta de equipo especializado.

La floración media de los maíces bajo estudio en el ciclo I fue más tardía 11 días que en el ciclo P-V. En la línea resistente varió de 84,8 a 90,5 días y en la susceptible de 81,3 a 113,1 días. La floración en la línea resistente se adelantó en promedio 16,6 días en los dos ciclos evaluados. Las aplicaciones de las tres concentraciones del aceite de A. indica adelantaron la floración aunque fue más precoz en la concentración de $20,8 \%$.

La altura de planta fue 23,6 cm mayor en el ciclo P-V en comparación al ciclo I. Si hubo diferencia significativa entre tratamientos para ésta variable. Las líneas susceptible y resistente tuvieron respectivamente 80,8 y $68,0 \mathrm{~cm}$. en promedio en ambos ciclos. El nim estimuló el crecimiento del maíz, aumento que se evidenció más en la línea susceptible, por ser la de menor porte.

El rendimiento de grano promedio en el ciclo P-V fue $107,2 \mathrm{~kg}^{\text {ha- }}{ }^{1}$ mayor en comparación al ciclo I, presentando la línea resistente una diferencia de 747,14 kg ha-1 en promedio con la línea susceptible. La concentración mayor $(20,8 \%)$ causó fitotoxicidad al cultivo, las dosis menores no, esto se vio reflejado en el rendimiento. 
La línea resistente con $A$. indica mostró menor daño que la línea susceptible tanto en I como en P-V (Figs. 1, 2 y 3). Al respecto Ayyangar y Rao (1989) indican que el nim no presenta de manera contundente efectos biocidas exclusivamente sobre $S$. frugiperda y $S$. littura, sino que actúa mas como repelente e inhibidor de la alimentación de larvas, causando así menor daño, es necesario realizar tantas aplicaciones como lo permita el ciclo de desarrollo del cultivo para cubrir el período de protección necesario contra el gusano cogollero.

Concentraciones con aceite de $\boldsymbol{A}$. indica. Con respecto a la floración (Fig. 10) se observó un adelanto de 33,8 días en promedio en la floración en el ciclo P-V respecto al ciclo I. Por otra parte, la línea resistente varió en promedio de 60,8 a 82,8 días y la susceptible de 61,3 a 117,8 días, por lo que la floración en la línea resistente se adelantó en promedio 16,6 días en los 2 ciclos evaluados. Respecto a la floración por ciclos de cultivo la línea resistente en P-V (61,3 días) se adelantó en promedio 17,4 días con respecto al ciclo I (78,7 días), en tanto que la línea susceptible en el ciclo P-V (61,6 días) se adelantó en promedio 50 días en comparación con el ciclo I (111,6 días). En cambio, la floración presentada en las 3 concentraciones del aceite de nim fue más rápida que el testigo (80,7 días); por ejemplo en la concentración 20,8\% de aceite de nim la floración se adelantó 7,4 días.

De manera general la aplicación de las tres concentraciones del aceite de A. indica $(0,2,2,0$ y 20,8\%), 4 horas antes de la infestación, 7 y a los 14 días después de la infestación artificial permite estructurar las siguientes inferencias: La concentración del 20,8\% protegió ligeramente más al maíz del daño de $S$. frugiperda. Esto es, puede existir compatibilidad de la aplicación del aceite de $A$. indica a la concentración del 20,8\% con la línea resistente (CML-67). Las aplicaciones de las tres concentraciones del aceite de $A$. indica adelantaron la floración aunque fue más precoz en la concentración del 20,8\%. La línea resistente fue más precoz en la floración que la línea susceptible. Las aplicaciones de las tres concentraciones del aceite de $A$. indica aumentaron la altura respecto al testigo, lo que sugiere que este producto estimula el crecimiento del maíz. Este aumento se evidenció más en la línea susceptible y no tanto en la resistente, por ser ésta de menor porte. La concentración de 20,8\% protegió ligeramente al maíz del daño de $S$. frugiperda, adelantó la floración y permitió mayor crecimiento del maíz, el rendimiento fue muy bajo, con un $37 \%$ de lo que fue el rendimiento del testigo, lo que muestra un efecto de fitotoxicidad. En cambio las concentraciones de 0,2 y 2,0\% con daño similar, floración precoz y mayor altura que el testigo permitieron el incremento del rendimiento respecto al testigo, en 39,2 y $13,5 \%$, respectivamente. Esto es explicado porque la dosis mayor causa fitotoxicidad al cultivo.

Para tener mejor desarrollo y mayor rendimiento de maíz el aceite de $A$. indica debe aplicarse a la concentración del $0,2 \%$ tres veces durante el período de 
crecimiento de 50 a 78 días después de la siembra. Las tres aplicaciones de $A$. indica a las concentraciones de $0,2,2,0$ y $20,8 \%$, en la etapa de crecimiento de 50 a 78 días del maíz son compatibles con la línea resistente, las mejores concentraciones son las de 0,2 y $2,0 \%$, debido a que la concentración mayor provoca fitotoxicidad.

AGRADECIMIENTOS. Los autores agradecen a Dr. Juan Rull sus valiosas aportaciones, dos revisores anónimos enriquecieron el manuscrito.

\section{LITERATURA CITADA}

Ayyangar, G.S.G. \& P.J. Rao. 1989. Neem (Azadirachta indica) (A.Juss) extracts as larval repellents and ovipositional deterrents to Spodoptera litura (Fabr). Indian Journal of Entomology, 51(2): 121124.

Iannacone-Oliver, J. A. \& Y. Murrugarra-Bringas. 2002. Efecto del nim y rotenona en las poblaciones de Tuta absoluta (Meyrick) (Lepidoptera: Gelechiidae) y en dos especies de áfidos (Homóptera: Aphididae) en el cultivo de tomate en Ica, Perú. Folia Entomologica Mexicana, 41(2): 119-128.

Kumar, H. 1994. Mechanisms of resistance in maize to Southwestern corn borer and Sugarcane borer. In: J.A. Mihm. (Ed.) Insect resistant maize. Recent advances and utilization. Proceedings of International Symposium held at CIMMYT. CIMMYT Batán, Edo. de México. pp. 54-55.

Levin-Mitchell, P., R. Gupta, A. A. Singh, \& P. Kumar. 2004. Behavioral and developmental effect of neen extracts on Clavigralla scutellaris (Hemiptera: Heteroptera: Coreidae) and its egg parasitoid Gryon fulviventre (Hymenoptera: Scelionidae). Journal Economic Entomology, 97(3): 916-923.

Mihm, J. A. 1983. Techniques for efficient mass rearing and infestation of fall armyworm, Spodoptera frugiperda J. E. Smith, for host plant resistance studies. Technical Bulletin. CIMMYT, México. 16 p.

Mihm, J. A. 1989. Evaluating maize for resistance to tropical stem borers, armyworms and earworms. pp. 109-121. In: J.A. Mihm and F. M. Davis (Eds.). Toward Insect Resistant Maize for the Third World. Proceedings of the international symposium on methodologies for developing host plant resistance to maize insects. CIMMYT. México D.F.

Mulla, M. S., \& T. Su. 1999. Activity and biological effects of neem products against arthropods of medical and veterinary importance. Journal of the American Mosquito Control Association, 15: 133-52.

Premachandra, D. W., C. Borgemeister, \& H. M. Poehling. 2005. Effects of neem and Spinosad on Ceratothripoides claratris (Thysanoptera: Thripidae) an important vegetable pest in Thailand. Journal Economic Entomology, 98(2): 438-448.

SAS Institute. 2000. SAS User's Guide: Statistics. Version 8.1 edition. SAalS Institute, Cary, NC. 1028 p.

Schmutterer, H. 1990. Properties and potential of natural pesticides from the neem tree, Azadirachta indica. Annual Review of Entomology, 35: 271-297.

Schmutterer, H. 1995. The neem tree Azadirachta indica A. Juss. and other meliaceous plants. VHC Publishers Inc., New York, USA. pp. 51-55.

Stocke, J. B., \& R. E. Redfern. 1982. Effect of sunlight on azadirachtin. Journal Environmental Science Health, 17: 57-65.

Williams, W. P., \& F. M. Davis. 1997. Mechanisms and bases of resistance in maize to southwestern corn borer and fall armyworm. In: J. A. Mihm (ed.), Proc. International Symposium. International Maize and Wheat Improvement Center. CIMMYT. México. 27 November-December 1994. CIMMYT. México, D. F. pp. 29-36.

Williams, W. P., F. M. Davis, P. M. Buckley, P. A. Hedin, G. T. Baker, \& D. S. Luthe. 1998. Factors associated with resistance to fall armyworm (Lepidoptera: Noctuidae) and southwestern corn borer 
Gutiérrez-García et al.: Efecto del nim en el daño por gusano cogollero en maíz

(Lepidoptera: Crambidae) in corn at different vegetative stages. Journal of Economic Entomology, 91: 1471-1480.

Yakkundi, S. R., Ch. S. S. R. Kumar, \& B. Ravindranath. 1996. Photochemistry of azadirachtin. $5^{\text {th }}$ International Neem Conference. Gatton, Australia. p. 21. 